\title{
Plasma-Based Surface Modification of Polydimethylsiloxane for PDMS-PDMS Molding
}

\author{
S. Lopera and R. D. Mansano \\ Laboratório de Sistemas Integráveis, Escola Politécnica University da São Paulo, São Paulo 05508-010, SP, Brazil
}

Correspondence should be addressed to S. Lopera, slopera@lsi.usp.br

Received 5 January 2012; Accepted 6 February 2012

Academic Editors: M. Patel and D. Pavel

Copyright (C) 2012 S. Lopera and R. D. Mansano. This is an open access article distributed under the Creative Commons Attribution License, which permits unrestricted use, distribution, and reproduction in any medium, provided the original work is properly cited.

\begin{abstract}
We present and compare two processes for plasma-based surface modification of Polydimethylsiloxane (PDMS) to achieve the antisticking behavior needed for PDMS-PDMS molding. The studied processes were oxygen plasma activation for vapor phase silanization and plasma polymerization with tetrafluoromethane/hydrogen mixtures under different processing conditions. We analyzed topography changes of the treated surfaces by atomic force microscopy and contact angle measurements. Plasma treatment were conducted in a parallel plate reactive ion etching reactor at a pressure of 300 mTorr, 30 Watts of RF power and a total flow rate of $30 \mathrm{sccm}$ of a gas mixture. We found for both processes that short, low power, treatments are better to create long-term modifications of the chemistry of the polymer surface while longer processes or thicker films tend to degrade faster with the use leaving rough surfaces with higher adherence to the molded material.
\end{abstract}

\section{Introduction}

As reviewed by Macdonald and Whitesides [1], PDMS (polydimethylsiloxane) has proven to be a suitable material for microfluidic device fabrication. It is optically transparent and can be molded to reproduce structures down to nanoscale resolution $[2,3]$, usually by direct casting on photoresist features over silicon wafers. SU8 is the most commonly used photoresist for master fabrication; single- or multilevel SU8 lines and structures have been extendedly used for PDMS microchannel definition, however, reutilization of resist masters is limited due to degradation by thermal stress during the curing process and by demolding forces. Some strategies such as coatings with diamond-like carbon (DLC) and DLC-polymer hybrids can be applied to increase the durability of the molds [4].

In this paper we present an improved method to replace the use of conventional photoresist masters with more durable positive copies in PDMS. Due to its elasticity, flexible masters in PDMS are not affected by thermal stress during the heating-cooling cycles of the curing process and it is easier to achieve a conformal contact between mold and countermold in closed molding configurations for the production of parts with through holes. Similar methods have been presented to mold complex 3D structures with through holes: Lucas et al. in 2008 [5] presented the use of flexible molds made with freestanding SU8 layers for double-side molding of PDMS, Jo et al. in 2000 [6] used a plastic sheet as countermold for SU8 molds and Janelle et al. in 2000 [7] presented the use of slabs of PDMS treated with threetridecafluorotetrahydrooctyl-trichlorosilane as countermolds.

Here we explore two plasma processes for surface modification of PDMS to achieve the antisticking behavior needed for PDMS-PDMS molding. For this application, three main characteristics are needed from the plasma treatment: the peeling force required to demold the PDMS parts off the treated molds should be low, the surface roughness of the mold should not be considerably increased, and the antisticking effect should be permanent and stable over the time.

\section{Materials and Methods}

2.1. Sample Preparation. We prepared PDMS samples using Sylgard 184 from Dow Corning, mixed in $1: 10$ proportion of curing agent to base polymer, degassed in vacuum for 
TABLE 1: Recipes for photolithography (SU8-2050).

\begin{tabular}{lccccc}
\hline Thickness & Spinning & Before bake & Exp. dose & After bake & Developing \\
\hline $50 \mu \mathrm{m}$ & $3000 \mathrm{RPM}$ & $95^{\circ} 15 \mathrm{~min}$ & $600 \mathrm{~mJ} \mathrm{~cm}^{-2}$ & $95^{\circ} 5 \mathrm{~min}$ & $5 \mathrm{~min}$ \\
$300 \mu \mathrm{m}$ & $500 \mathrm{RPM}$ & $95^{\circ} 4$ hours & $3600 \mathrm{~mJ} \mathrm{~cm}^{-2}$ & $95^{\circ} 15 \mathrm{~min}$ & $30 \mathrm{~min}$ \\
\hline
\end{tabular}

$30 \mathrm{~min}$ and oven baked at $100^{\circ} \mathrm{C}$ for 30 minutes after casting over silicon wafers. We prepared also PDMS samples with rough surfaces, by casting on the rear face of a silicon wafer to increase the contact area for adhesion tests. All PDMS pieces were cast with a thickness of approximately $2 \mathrm{~mm}$, cut in $2 \mathrm{~cm} \times 2 \mathrm{~cm}$ squares and plasma bonded to $1^{\prime \prime} \times 3^{\prime \prime}$ glass slides for easy handling. The samples were rinsed with distilled water, sonicated in isopropyl alcohol for 5 minutes, and rinsed again in distilled water to remove particles prior to the treatments.

2.2. Fluorinated Plasma Treatments. This treatment consists in a plasma polymerization of fluorinate compounds over the PDMS surface using $\mathrm{CF}_{4} / \mathrm{H}_{2}$ mixtures. Two different $\mathrm{CF}_{4} / \mathrm{H}_{2}$ proportions were studied: $5 \%$ hydrogen and 20\% hydrogen with process times of 1,5 , and 10 minutes. Fluorinated plasma treatments were conducted in a parallel plate RIE reactor at a pressure of $300 \mathrm{~m}$ Torr, 30 Watts of RF power $(13.56 \mathrm{MHz})$, and a total flow rate of $30 \mathrm{sccm}$ of the desired gas mixture.

2.3. Plasma-Activated Silanization. The second method consists in an oxygen plasma activation of the PDMS surface to promote de incorporation of Hexamethyldisiloxane molecules. PDMS samples were plasma oxidized in a remote plasma reactor to avoid direct bombardment of the surface. This reactor is composed by a $1^{\prime \prime}$ quartz tube, $20 \mathrm{~cm}$ long, with an oxygen inlet at one end connected to a vacuum chamber containing the samples at the other end. High DC voltage is applied between the ends to produce a glow discharge at $300 \mathrm{~m}$ Torr $18 \mathrm{~W}$ of DC power for 50 seconds and placed on a hotplate at $100^{\circ} \mathrm{C}$ with two drops of HMDS, covered with a $6^{\prime \prime}$ Petri dish immediately afterward.

2.4. Surface Topography and Adhesion to PDMS. The surface topography of the processed samples was analyzed by atomic force microscopy using a NanoSurf Nanite AFM. We did also contact angle measurements with a simple imaging setup and using the low-bond axisymmetric drop shape analysis (LBADSA) script for Image J developed by Stalder et al. [8].

The adhesive forces of the different treated surfaces to the PDMS were qualitatively compared by manual pealing straps of PDMS cured over each sample, ordering form low to high the force required to peal the PDMS apart. The surface interactions with PDMS were also studied by atomic force spectroscopy (AFS), with a contact-mode AFM tip (NanoWorld CNTR-10) coated with PDMS.

2.5. Flexible Molds Fabrication. To illustrate the application of the studied antisticking treatments for the fabrication of flexible PDMS copies of micromolds, we fabricated two level
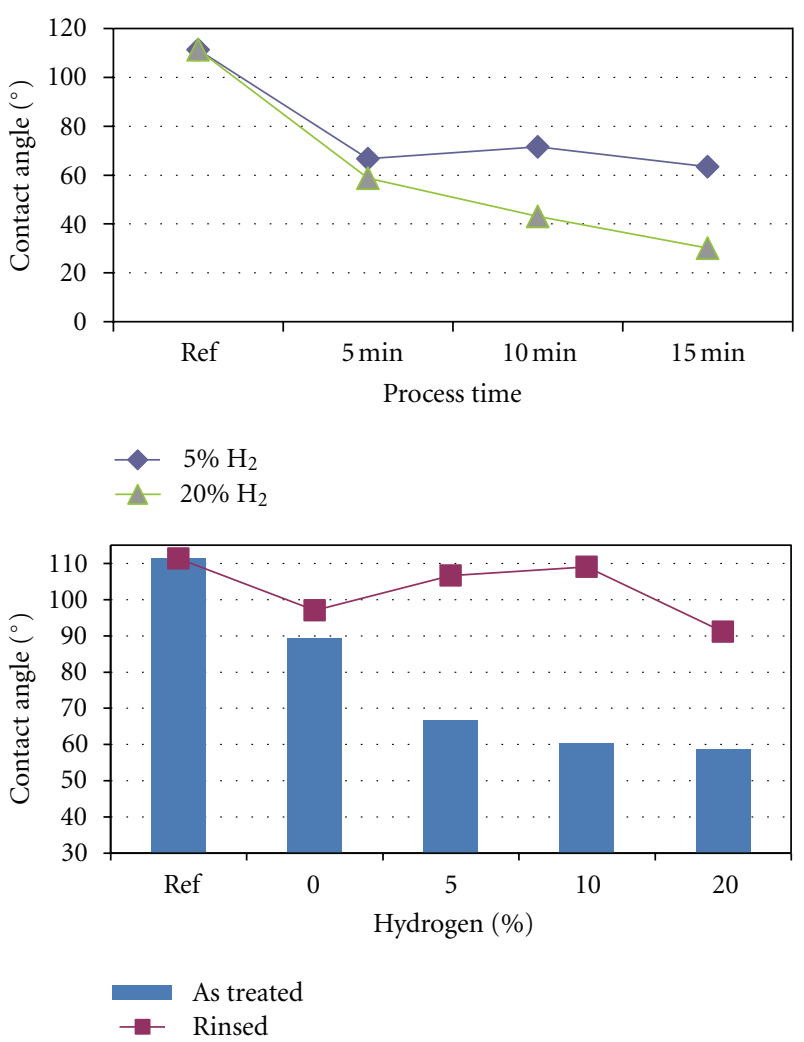

Figure 1: Contact angle of PDMS samples treated with $\mathrm{CF}_{4} / \mathrm{H}_{2}$ under different conditions.

SU8 masters onto $3^{\prime \prime}$ glass wafers by using high resolution photoplotted sheet as a mask. The first layer was $50 \mu \mathrm{m}$ thick, to define channels and the second layer $300 \mu \mathrm{m}$ thick for interconnection posts. Table 1 summarizes the lithographic recipes for each layer.

The flexible molds are obtained in a two steps process, first we use the SU8/silicon mold to cast a PDMS inverted copy that is then treated with the selected antisticking plasma process and used to cast the positive copy in PDMS that is also plasma treated for further use.

\section{Results and Discussion}

3.1. Contact Angle Measurements. In a preliminary study we had observed that the contact angle of the PDMS samples to water decreases with the plasma processing time and with the hydrogen percentage in the gas mixture, as shown in Figure 1, but the hydrophobicity of the samples is partially recovered after rinsing with water. 


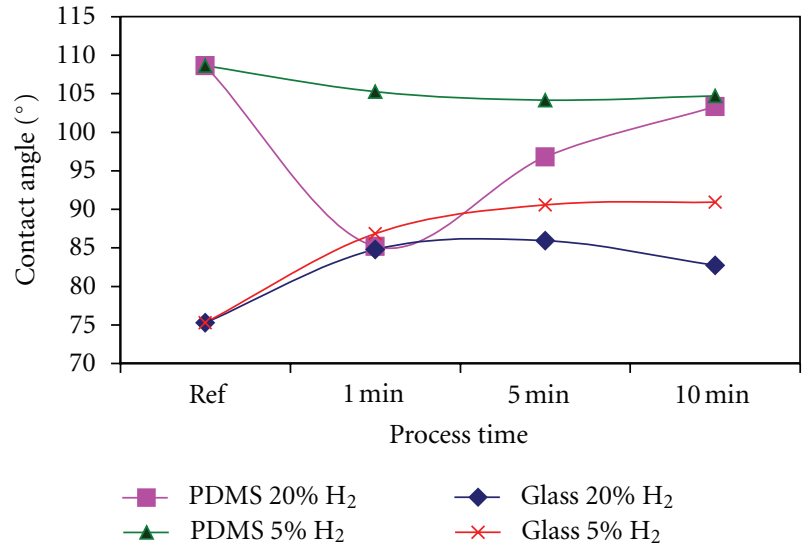

FIGURE 2: Contact angle of PDMS and glass samples processed with $\mathrm{CF}_{4} / \mathrm{H}_{2}$ plasmas at $300 \mathrm{~m}$ Torr $30 \mathrm{~W}$.

The hydrophilic behaviour of the treated samples can be explained by three possible factors: an increment in the surface roughness, the polymerization of a less hydrophobic film on the surface, or the production of polar or charged terminations in the PDMS chains. As the surfaces became again hydrophobic after rinsing with water it is plausible that instable polar groups be primarily responsible for the measured low contact angles and this groups are removed by the dipole interactions with the water.

The curves in Figure 2 correspond to the contact angles measured on PDMS and glass of the treated samples after rinsing for 10 minutes with IPA and water to remove nonbonded compounds. On glass, the fluorinated plasma treatment has the effect of increasing the contact angle while on PDMS the treatment decreases the contact angle what indicates that the deposited polymer has an intermediate contact angle around $90^{\circ}$. The results of both treatment with $5 \%$ hydrogen and $20 \%$ hydrogen are similar on glass but very dissimilar on the PDMS, this occurs by the depletion of fluorine ions by the etching reactions with the glass that increase locally the relative proportion of hydrogen, getting close to the saturation of polymerization rate observed after $20 \%$ of hydrogen.

Comparing the evolution of the contact angle of the "as treated" samples with the process time (Figure 1) and the same for samples after rinsing with IPA (Figure 2), it is evident that the rinsing process degrades somehow the polymerized film, recovering partially the original contact angle value of the original substrate.

From Figure 2 it is also noted that thicker films, deposited with $20 \%$ hydrogen and longer times, are more affected by the rinsing process and that the adherence of the fluorinated polymer to the glass substrates seems to be stronger than the adherence to PDMS substrates.

3.2. Antisticking Behavior. All the studied treatments conferred to the PDMS in major or minor degree the desired antisticking effect and it was possible to classify from low to high the peeling force required to manually lift with tweezers a $200 \mu \mathrm{L}$ drop of PDMS cured on each treated surface. Seven

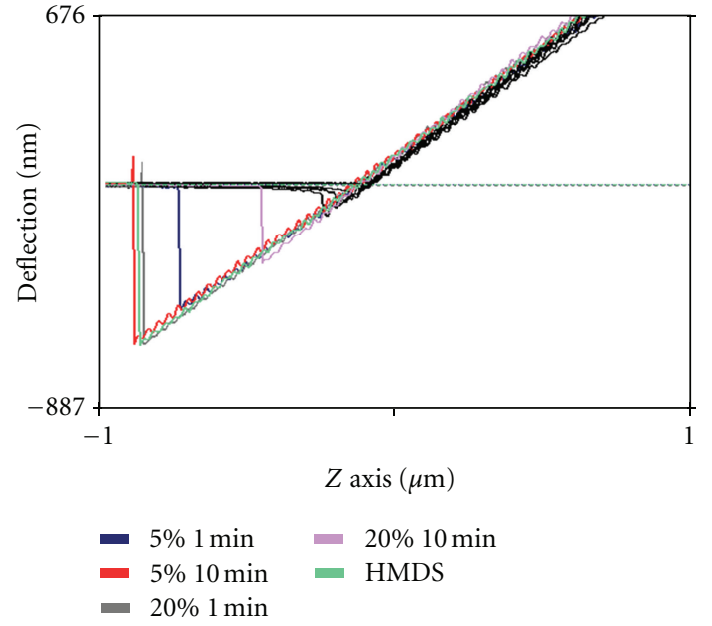

(a)

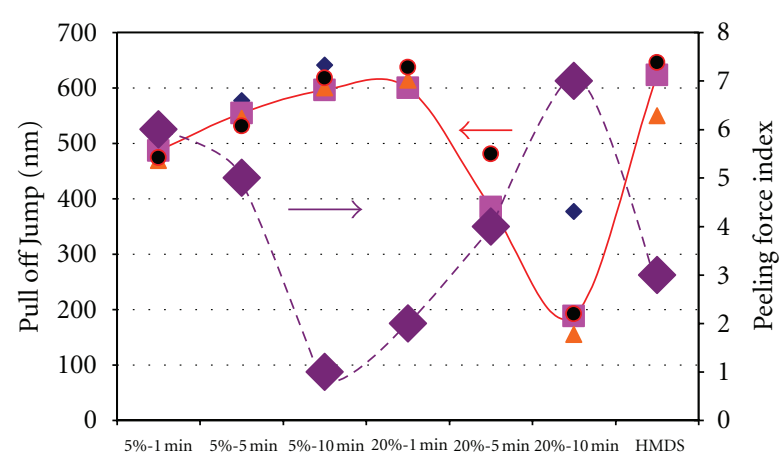

Treatment

(b)

Figure 3: Atomic force spectroscopy curves (a) and AFS pull-off jump (b).

samples corresponding to two $\mathrm{CF}_{4} / \mathrm{H}_{2}$ proportions for three processing times plus the silanized PDMS sample were then indexed from 1 to 7 according to the qualitative comparison made by two experimenters in three independent repetitions.

The atomic force spectrograms of the samples exhibited different pull-off jumps between treatments and also different point to point variability within each sample. In Figure 3 we plotted the different AFS spectrograms. It was expected that the pull-off jump, which is the maximum negative deflection of the AFM cantilever before it loses contact with the sample in the backward part of the AFS cycle, would be proportional to the adhesive force of the PDMS to the treated surface, however when we plotted together the 1-7 index of the peeling force and the AFS data of the seven samples the results were completely inverse; the graph in Figure 4 at the right shows this superimposed data plots where the lower pull off jump corresponded to the higher peeling force and vice versa.

This apparent inconsistency could be related to the surface roughness that can affect the effective contact area between the coated tip and the sample; if the surface of a sample is so rough that only a few peaks of its topography 


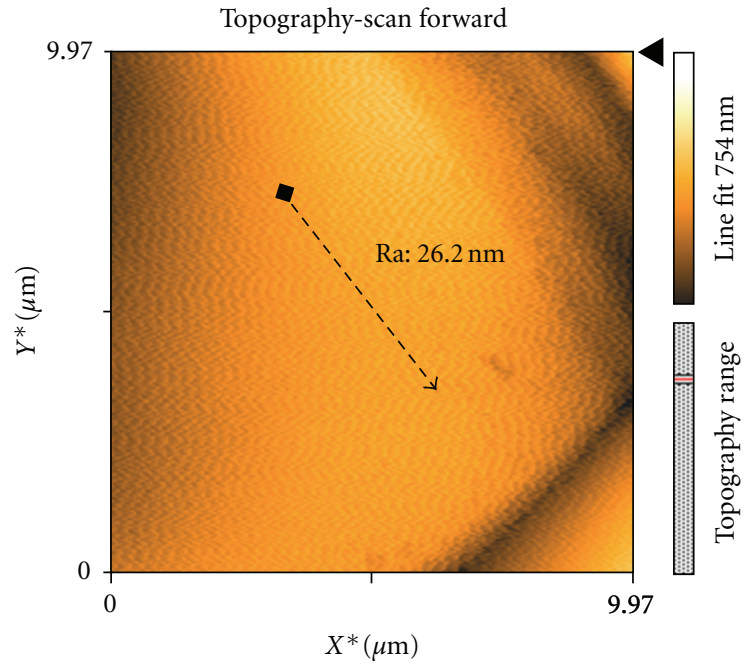

Un-treated PDMS reference

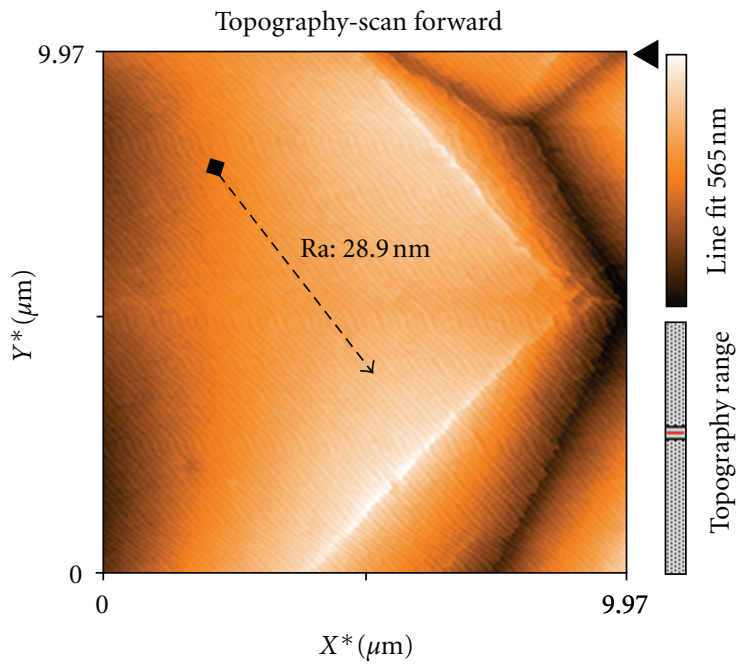

$1 \mathrm{~min}$ of $\mathrm{CF}_{4}+20 \%$

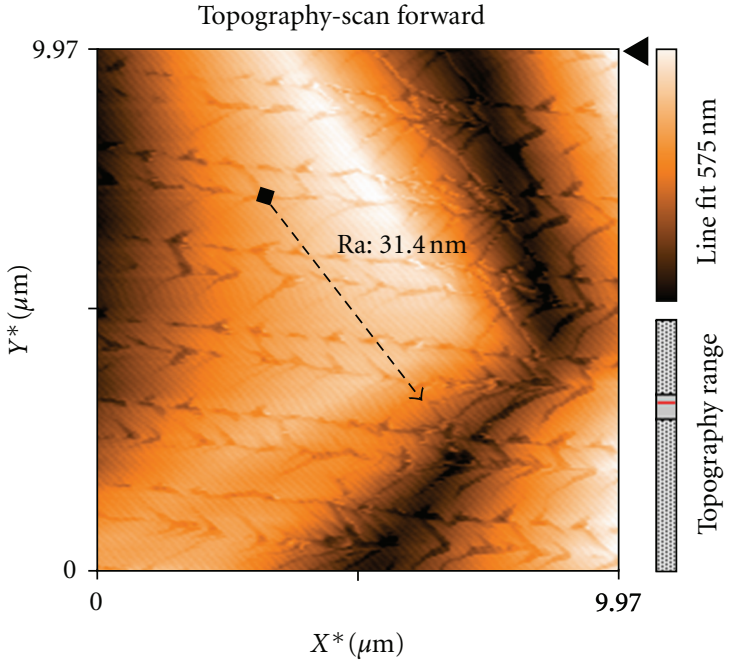

Silanized surface (HMDS)

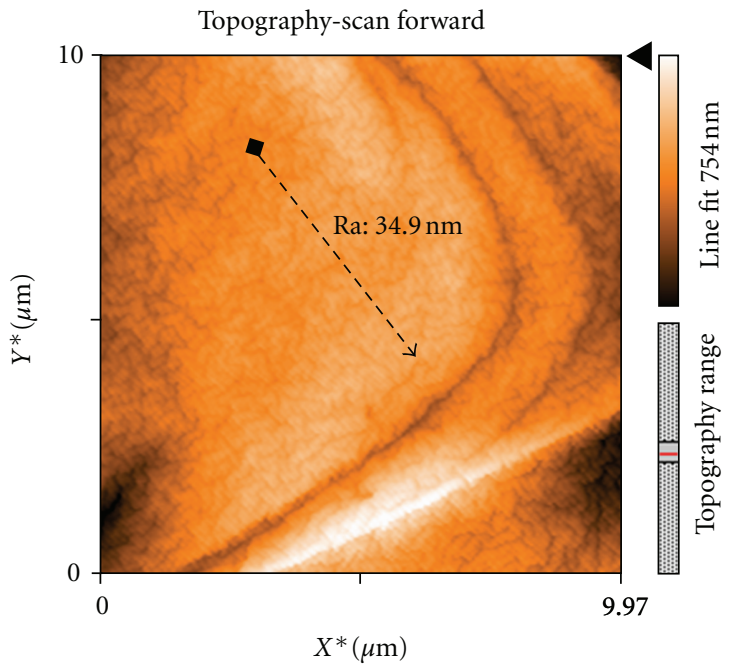

$10 \min$ of $\mathrm{CF}_{4}+20 \%$

Figure 4: AFM images of the PDMS samples: untreated PDMS reference, silanized surface, and $\mathrm{CF}_{4}+20 \% \mathrm{H}_{2}$ for 1 and 10 minutes.
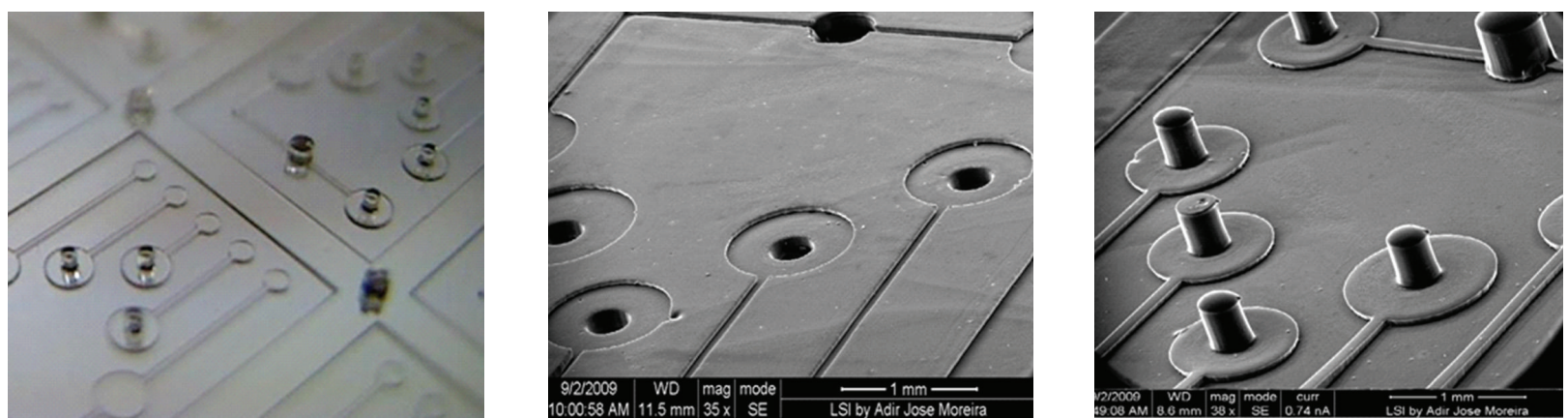

FIgURE 5: SU8 mold (left), negative copy in PDMS (center), and positive copy (right).

touch the coated AFM tip then the measured pull of force will be low, but on the contrary the effective contact area with a PDMS drop cured on it will be higher requiring a bigger force to peel it off.
The samples with higher variability that correspond to the lower pull off forces are also the samples with thicker polymerized films (20\% hydrogen, 5 and $10 \mathrm{~min})$ that appeared to be degraded during the rinsing, this reaffirms 
that in some places the film could be away giving different measurements from point to point. The AFM images of the surface topography can give more information on this topic.

3.3. Surface Topography. In Figure 4 we present the AFM images of some treated surfaces and the untreated PDMS reference. The three samples processed with 5\% hydrogen and the sample for one minute process with $20 \%$ hydrogen showed a surface quality similar to the untreated reference, all of them remained optically clear with a slight increase in roughness; only the samples with longer treatments for higher hydrogen concentration produced thicker brown films that led to a rough texture after rinsing.

These results are consistent with the previous observations of AFS and contact angle measurements, indicating that thinner films are more stable than thicker films. The HMDS silanization formed a cracked-like texture on the samples but with a low main roughness.

Comparing all the studied treatments we found very similar results for 1 minute $\mathrm{CF}_{4}+20 \%$ hydrogen and 10 minutes $\mathrm{CF}_{4}+5 \%$ hydrogen what could be associated with a similar film thickness, both having low peeling forces, low roughness and the higher measured AFS pull-off jump. The silanization process showed also similar AFS values and a low peeling force, however, with a characteristic texture that could be problematic for some applications.

3.4. PDMS-PMDS Molding. In Figure 5 we present optical and electronic micrographs of a micromold and its negative and positive copies obtained in PDMS with the proposed replication technique. Demolding PDMS parts from this flexible copies was found to be even easier that from the original SU8 Mold.

\section{Conclusion}

All studied treatments achieved the desired antisticking effect on PDMS surfaces, however long plasma treatments increase the surface roughness and create thicker films that are delaminated by rinsing or demolding forces losing its effect. The best results were obtained with 1 min treatment $\mathrm{CF}_{4}+20 \%$ $\mathrm{H}_{2}, 10$ min treatment $\mathrm{CF}_{4}+5 \% \mathrm{H}_{2}$, and plasma-activated silanization, all of them requiring low peeling forces and having a good surface quality.

We demonstrated the applicability of the developed method for the fabrication of flexible copies of SU8 masters for microfluidics.

\section{References}

[1] J. C. McDonald and G. M. Whitesides, "Poly(dimethylsiloxane) as a material for fabricating microfluidic devices," Accounts of Chemical Research, vol. 35, no. 7, pp. 491-499, 2002.

[2] J. Lee, S. Park, K. Choi, and G. Kim, "Nano-scale patterning using the roll typed UV-nanoimprint lithography tool," Microelectronic Engineering, vol. 85, no. 5-6, pp. 861-865, 2008.

[3] F. Hamouda, G. Barbillon, S. Held et al., "Nanoholes by soft UV nanoimprint lithography applied to study of membrane proteins," Microelectronic Engineering, vol. 86, no. 4-6, pp. 583585, 2009.

[4] M. Okada, M. Iwasa, K. I. Nakamatsu et al., "Evaluation of fluorinated diamond like carbon as antisticking layer by scanning probe microscopy," Journal of Photopolymer Science and Technology, vol. 21, no. 4, pp. 597-599, 2008.

[5] N. Lucas, S. Demming, A. Jordan, P. Sichler, and S. Büttgenbach, "An improved method for double-sided moulding of PDMS," Journal of Micromechanics and Microengineering, vol. 18, no. 7, Article ID 075037, 2008.

[6] B. H. Jo, L. M. Van Lerberghe, K. M. Motsegood, and D. J. Beebe, "Three-dimensional micro-channel fabrication in polydimethylsiloxane (PDMS) elastomer," Journal of Microelectromechanical Systems, vol. 9, no. 1, pp. 76-81, 2000.

[7] R. A. Janelle, D. T. Chiu, R. J. Jackman et al., "Fabrication of topologically complex three-dimensional microfluidic systems in PDMS by rapid prototyping," Analytical Chemistry, vol. 72, no. 14, pp. 3158-3164, 2000.

[8] A. F. Stalder, T. Melchior, M. Müller, D. Sage, T. Blu, and M. Unser, "Low-bond axisymmetric drop shape analysis for surface tension and contact angle measurements of sessile drops," Colloids and Surfaces, A, vol. 364, no. 1-3, pp. 72-81, 2010. 

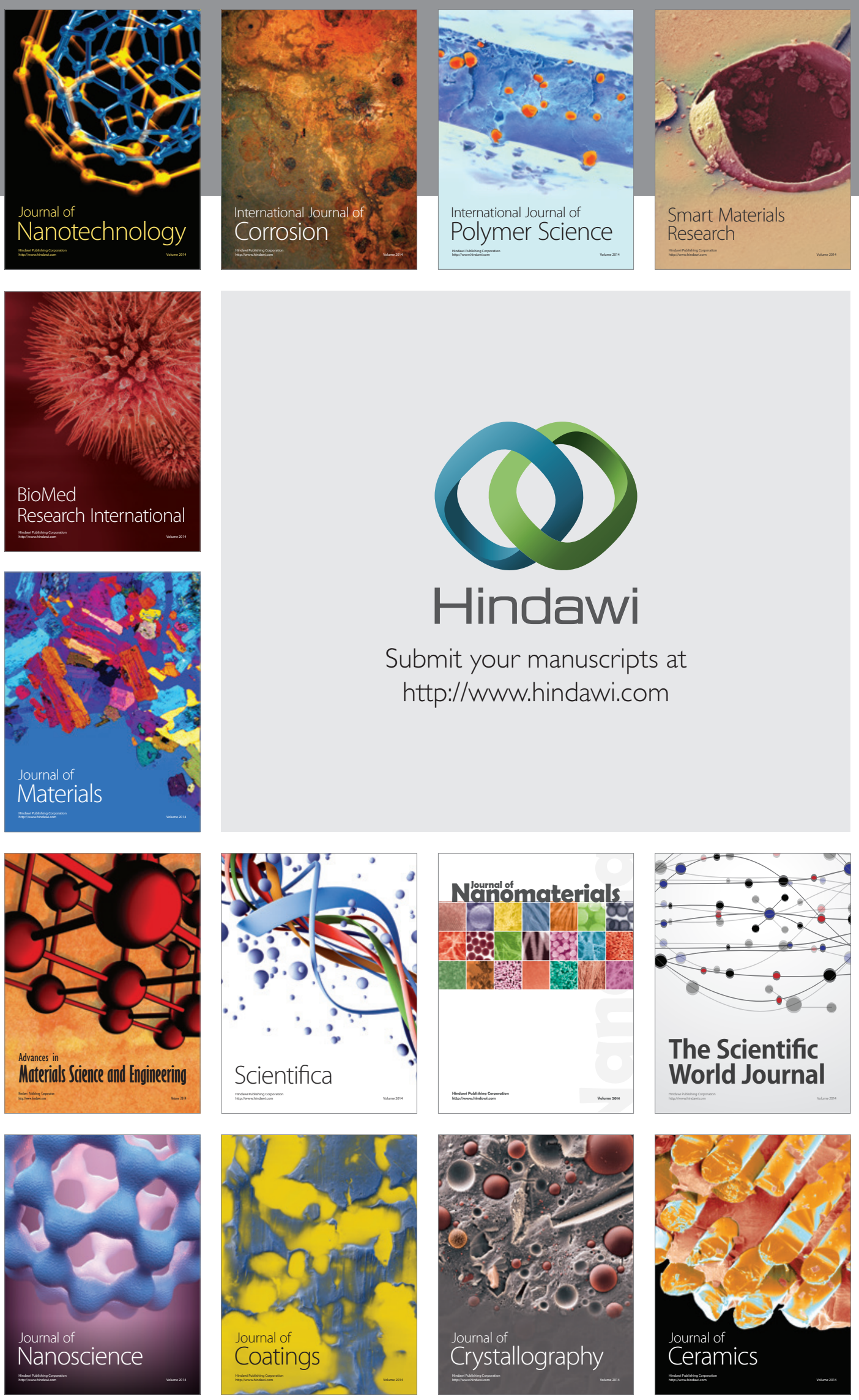

The Scientific World Journal

Submit your manuscripts at

http://www.hindawi.com

\section{World Journal}

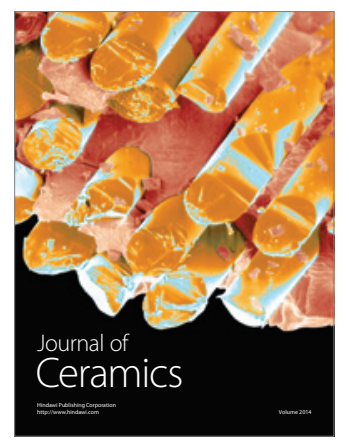

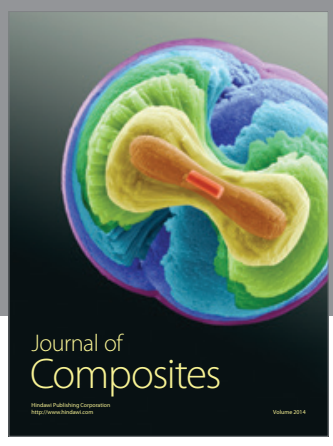
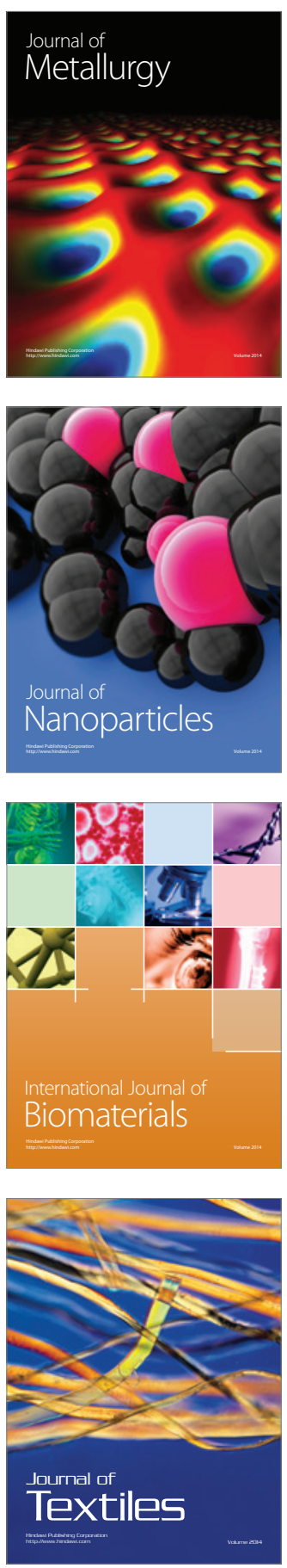